\title{
Detection of antibodies to peste-des-petits-ruminants virus in the semi-domesticated yak
}

\author{
Muhammad Abubakar ${ }^{1}$ - Nosirjon Sattorov ${ }^{2}$. Shumaila Manzoor ${ }^{3}$ - Ehtisham ul Haq Khan ${ }^{3} \cdot$ Manzoor Hussain $^{3}$. \\ Aamir Bin Zahur ${ }^{1} \cdot$ Muhammad Afzal $^{3}$. Jonas Johansson Wensman ${ }^{4}$ (C)
}

Received: 11 January 2019 / Revised: 9 August 2019 / Accepted: 21 September 2019 /Published online: 4 Novvember 2019

(C) The Author(s) 2019

\begin{abstract}
Peste des petits ruminants (PPR) is a highly contagious and acute viral disease of small ruminants. Occasionally, PPR can affect wildlife with devastating results, such as the recent outbreaks in Mongolian saiga. The yak (Bos grunniens and Bos mutus) is a large ruminant found throughout the Himalayan region of south Central Asia, the Tibetan Plateau and as far north as Mongolia and Russia. In Pakistan, yaks are confined to the high plateau of the Northern Areas, from Gilgit to the valley of Ladakh. In Tajikistan, yaks are present in districts in the north, east and southeast of the country. Commonly, yak intermingle with domestic animals during winter when mountainous summer pastures are covered with snow, and with wildlife during summer pastures. PPR is considered endemic in sheep and goats in Pakistan and Tajikistan. In this study, we investigated the potential presence of antibodies to peste-des-petits-ruminants virus (PPRV) in yak populations using ELISA. A total of 250 (Pakistan) and 85 (Tajikistan) serum samples of healthy yaks were collected. None of the Tajik yaks were seropositive $(95 \%$ confidence interval (CI) $0.0-4.2 \%$ ), while 23 of 250 (9.2\%; 95\% CI 5.9-13.5\%) yaks sampled in Pakistan were found positive. Whether PPRV is continuously circulating among yaks or seroconversion reflects spill-over from outbreaks of PPR in domestic animals remains unknown. Due to the herding practices, yak might transmit PPR from domestic to wild ruminants. Differences in contacts between yaks and domestic animals in Pakistan and Tajikistan, or the low sample size, could explain that no Tajik yaks were seropositive.
\end{abstract}

Keywords Morbillivirus $\cdot$ Epidemiology $\cdot$ Serology $\cdot$ Sheep $\cdot$ Goats $\cdot$ Wildlife

The yak (Bos grunniens and Bos mutus) is a long-haired bovine found throughout the Himalayan region of south Central Asia, the Tibetan Plateau and as far north as Mongolia and Russia. Yak can be classified as wild, semi-domestic and domestic. In Pakistan, yaks are confined to the high plateau of the Northern Areas, from Gilgit to the valley of Ladakh, with an estimated population of 14,900 (Khan 1996). In Tajikistan, yaks are kept in three areas: in the northern Sharihston district $(n=200$; occasionally these animals are using a shared pasture with Ayni district), in the eastern Murghob district of the
Jonas Johansson Wensman

jonas.wensman@slu.se

Muhammad Abubakar

mabnvl@gmail.com

Nosirjon Sattorov

sattorov_n@inbox.ru

Shumaila Manzoor

shumaila.manzoor@fao.org

Ehtisham ul Haq Khan

ehtisham26@yahoo.com

Manzoor Hussain

manzoor.hussain@fao.org
Aamir Bin Zahur

aamerabz@yahoo.com

Muhammad Afzal

muhammadimam.afzal@fao.org

National Veterinary Laboratory, Park Road, Islamabad, Pakistan

2 Center for National Collection of Pathogenic Microorganisms, Institute of Biosafety Problems, Tajik Academy of Agricultural Sciences, Dushanbe, Tajikistan

3 Progressive control of Foot and Mouth Disease in Pakistan (GCP/ PAK/123/USA), FAO National Project, Islamabad, Pakistan

4 Department of Clinical Sciences, Swedish University of Agricultural Sciences, P.O. Box 7054, SE-750 07 Uppsala, Sweden 
Pamir province $(n=17,000)$, and in the southeastern Shurobod district $(n=100)$. Yaks are important assets for the livelihood of people in these areas. They are kept for meat as well as for milk and wool production. Yaks are mainly kept as semi-domesticated, where they come near human populations in winter to get shelter and feed and share grazing and watering areas with domestic animals (Hashmi et al. 2017). During the summer, yaks are migrating to summer pastures in mountainous areas on high altitude, where intermingling with wildlife occur.

Peste des petits ruminants (PPR) is a highly contagious and acute viral disease of mainly small ruminants, such as goats and sheep, that also can affect wild animals (Aziz-Ul-Rahman et al. 2018; Munir 2014). The disease is caused by peste-des-petits-ruminants virus (PPRV), classified as a Small ruminant morbillivirus (Amarasinghe et al. 2018). Recently, PPR has caused devastating outbreaks among the endangered Mongolian saiga (Saiga tatarica mongolica) and is considered a threat to wildlife conservation (Aguilar et al. 2018). Presence of PPR has been recognised in Pakistan since 1991 (Abubakar and Munir 2014), and the first outbreak of PPR in Tajikistan was reported in 2004 (Kwiatek et al. 2007). The disease is now considered endemic in the sheep and goat populations of these countries (Abubakar and Munir 2014; Shonazarov 2012). Bovines, such as cattle and buffaloes, can be infected by PPRV, resulting in subclinical infection (cattle and African buffaloes; Syncerus caffer; (Albina et al. 2013)) or in some cases clinical signs of PPR (water buffaloes; Bubalus bubalis) (Govindarajan et al. 1997). Previous studies from China have indicated yak to seroconvert to PPRV. During a PPR outbreak among goats in Tibet, nine yaks without clinical signs were sampled (Zhao et al. 2009). One of the yaks had seroconverted, most likely as a result of spill-over from the outbreak in goats. A seroprevalence study of yak in various regions of China indicated an overall seroprevalence of $11.2 \%$, with a higher seroprevalence in Tibet (bordering to Pakistan) and Sichuan (Li et al. 2018). In this study, the aim was to investigate the presence of antibodies to PPRV in yaks in Pakistan and Tajikistan, where PPR is endemic in domestic animals.

A total of 250 serum samples of apparently healthy yaks were collected from four different localities of Gilgit-Baltistan (GB), Pakistan in October-November 2014 (early winter) (Fig. 1; Table 1). In Tajikistan, 85 apparently healthy yaks over 2 years of age (13 females, 67 males and in 5 animals, sex was not recorded) were sampled during June-August 2016 in the districts of Sharihston $(n=22)$, Ayni (bordering to Sharihston, and yaks originating from Sharihston were using a shared pasture; $n=13)$ and Murghob $(n=50)$ (Fig. 1). In all sampling areas, yak intermingled with domestic animals (including sheep and goats) and wildlife, depending on season. Serum samples were heat inactivated before being analysed in duplicate according to the manufacturer's instructions using a commercial competitive ELISA (ID-screen PPR competition ELISA, IDVet, Grabels, France) detecting antibodies specific to the nucleoprotein of PPRV (Libeau et al. 1995). Statistical analysis was performed using a Chi-square test with a $p$ value of 0.05 considered significant, and $95 \%$ confidence intervals were calculated.

None of the sampled Tajik yaks were found seropositive (95\% CI $0.0-4.2 \%$ ), whereas 23 out of 250 serum samples from yaks in Pakistan $(9.2 \%$; 95\% CI 5.9-13.5\%; Table 1) were found positive for PPRV antibodies. At the time of sampling, no active clinical outbreak was reported in this area. A higher proportion of animals older than 2 years of age was seropositive compared with animals $\leq 2$ years $(11.9 \%$ vs. $6.1 \% ; p=0.116$; Chi-square test), although this was not considered statistically significant. Presence of seropositive
Fig. 1 Map of Tajikistan and Pakistan with the sampling areas indicated in red. A-Shahriston, Tajikistan. B-Ayni, Tajikistan. C-Murghob, Tajikistan. DGilgit-Baltistan (GB), Pakistan. The yellow marker indicates the sampling sites (Ghizer and Phundar) in Ghizer district, where PPRV seropositive sera have been found. Map contributed by Emeli Torsson, Swedish University of Agricultural Sciences

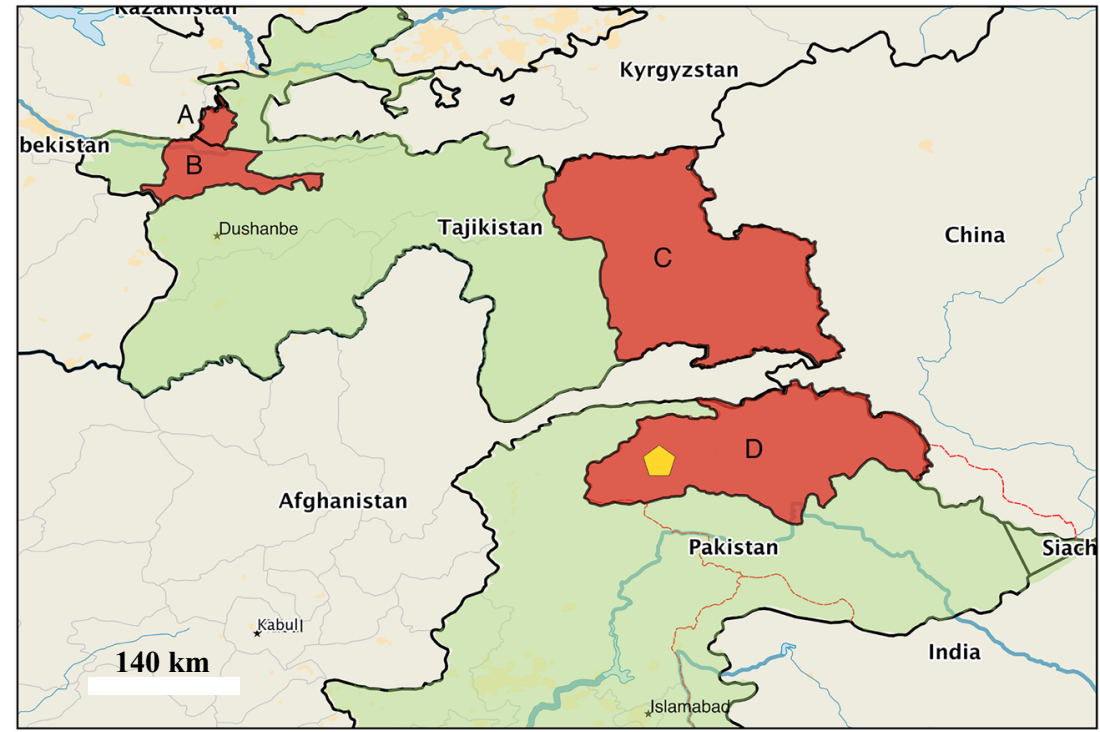


Table 1 Seropositivity to PPRV for yaks sampled in Pakistan

\begin{tabular}{lll}
\hline Variable & Analysed (positive) & $\%$ positive $(95 \%$ CI) \\
\hline Total & $250(23)$ & $9.2(5.9-13.5)$ \\
Female & $155(17)$ & $11.0(6.0-15.9)$ \\
Male & $95(6)$ & $6.3(1.4-11.2)$ \\
$\leq 2$ years & $115(7)$ & $6.1(2.5-12.1)$ \\
$>$ 2 years & $135(16)$ & $11.9(6.9-18.5)$ \\
Phundar area 1 & $58(5)$ & $8.6(2.9-19.0)$ \\
Phundar area 2 & $64(8)$ & $12.5(5.6-23.2)$ \\
Ghizer area 1 & $67(7)$ & $10.4(4.3-20.3)$ \\
Ghizer area 2 & $61(3)$ & $4.9(1.0-13.7)$ \\
\hline
\end{tabular}

animals in all four localities in GB indicates that PPR is widespread in the area. A higher proportion of females was positive for PPR in comparison with males (11.0\% vs. $6.3 \%$; $p=$ 0.217 ; Chi-square test), although this was not statistically significant.

Whether PPRV is continuously circulating among yaks or seroconversion reflects spill-over from outbreaks of PPR in domestic animals remains unknown. Overall seroprevalence in goats sampled in 2005-2006 in GB was estimated at $24.0 \%$, with a lower seroprevalence in Ghizer district (14.5\%) (Abubakar et al. 2017). During the period 20052007, three PPR outbreaks in GB were reported (Zahur et al. 2014). At the time of sampling, no active clinical outbreak in GB was reported, but a few months later, an outbreak was reported, indicating the ongoing circulation of PPRV in this area. Especially during winter, yaks intermingle with domestic animals and could then be exposed to PPRV excreted by infected sheep and goats. Differences in contact patterns between yaks and domestic animals in Pakistan and Tajikistan, or the low sample size, could explain that Tajik analysed yaks were not seropositive. A recently reported study on yaks in neighbouring Tibet showed that males were more likely to be seropositive than females (approx. 25\% in males vs. $10 \%$ in females), with an increasing proportion of seropositive animals due to age (Li et al. 2018), reflecting the higher chance of being exposed to PPRV with rising age. In our study, there was similarly a tendency for animals over 2 years of age to more likely be seropositive, but in contrast female yaks had a higher proportion of seropositivity than male yaks $(11 \%$ in female yaks vs. $6.3 \%$ in male yaks), although this was not statistically significant. Overall, $15.5 \%$ (95\% CI $11.7-$ 19.9\%) of Tibetan yaks were seropositive for PPR (Li et al. 2018), while in GB, Pakistan, the seroprevalence was significantly lower $(9.2 \%$; 95\% CI $5.9-13.5 \% ; p<0.05$; Chi-square test); however, the same ELISA was not used in the two studies, which could influence these results. Tibet was the first region in China to encounter a PPR outbreak in 2007 with a seroprevalence of $34.1 \%$ in goats (Liu et al. 2018). Due to a successful stamping out, no PPR outbreaks were reported in
Tibet after 2010. The study on yak in Tibet does unfortunately not state which year the sampling occurred ( $\mathrm{Li}$ et al. 2018). Thus, it is unclear whether the Tibetan yaks seroconverted during the period of active PPR outbreaks, as a spill-over effect, or if it reflects a potential silent virus circulation within the yak or other animal populations. It is also not known whether yak can develop clinical signs of PPR. Other bovines are commonly subclinically infected (Albina et al. 2013), and yaks investigated during a PPR outbreak did not develop clinical signs (Zhao et al. 2009). Variation in susceptibility to PPRV infection between species is probably due to host receptor affinity (Kumar et al. 2014), as suggested by the fact that Vero cells expressing the goat signalling lymphocyte activation molecule (SLAM) receptor are more prone to PPRV infection than cells expressing the bovine SLAM receptor (Adombi et al. 2011). Circulation of PPRV in domestic animals intermingling with wildlife has been shown to be a threat to endangered wildlife species (Aziz-ul-Rahman et al. 2018), such as the Mongolian saiga (Aguilar et al. 2018). Semidomestic yaks are intermingling with both domestic and wild animals, depending on season, and might therefore be a potential source for such spill-over effects from domestic animals to wildlife. Although other bovine species are considered dead-end-hosts for PPRV, investigations are needed to elucidate if yak could play a role in disease transmission to domestic and wild animals.

Acknowledgements The authors would like to thank Dr. Muhammad Munir, Lancaster University, UK and Dr. Giorgi Metreveli for valuable discussions and Emeli Torsson, Swedish University of Agricultural Sciences, for her contribution with the map of Tajikistan and Pakistan.

Funding information Open access funding provided by Swedish University of Agricultural Sciences. This study was funded by the Swedish Research Council (grants no. 348-2013-6402 and 348-20144293).

\section{Compliance with ethical standards}

Conflict of interest The authors declare that they have no conflict of interest.

Open Access This article is distributed under the terms of the Creative Commons Attribution 4.0 International License (http:// creativecommons.org/licenses/by/4.0/), which permits unrestricted use, distribution, and reproduction in any medium, provided you give appropriate credit to the original author(s) and the source, provide a link to the Creative Commons license, and indicate if changes were made.

\section{References}

Abubakar M, Munir M (2014) Peste des petits ruminants virus: an emerging threat to goat farming in Pakistan. Transbound Emerg Dis 61(Suppl 1):7-10. https://doi.org/10.1111/tbed.12192 
Abubakar M, Bin Zahur A, Afzal M, Ali Q, Gonzales J (2017) Peste des petits ruminants (PPR) in Pakistan: analysis of national level of serological data. Small Rumin Res 155:57-65. https://doi.org/10. 1016/j.smallrumres.2017.08.017

Adombi CM et al (2011) Monkey CV1 cell line expressing the sheep-goat SLAM protein: a highly sensitive cell line for the isolation of peste des petits ruminants virus from pathological specimens. J Virol Methods 173:306-313. https://doi.org/10.1016/j.jviromet.2011.02.024

Aguilar XF, Fine AE, Pruvot M, Njeumi F, Walzer C, Kock R, Shiilegdamba E (2018) PPR virus threatens wildlife conservation. Science 362:165-166. https://doi.org/10.1126/science.aav4096

Albina E, Kwiatek O, Minet C, Lancelot R, Servan de Almeida R, Libeau $\mathrm{G}$ (2013) Peste des petits ruminants, the next eradicated animal disease? Vet Microbiol 165:38-44. https://doi.org/10.1016/j. vetmic.2012.12.013

Amarasinghe GK et al (2018) Taxonomy of the order Mononegavirales: update 2018. Arch Virol 163:2283-2294. https://doi.org/10.1007/ s00705-018-3814-x

Aziz-ul-Rahman, Wensman JJ, Abubakar M, Shabbir MZ, Rossiter P (2018) Peste des petits ruminants in wild ungulates. Trop Anim Health Prod. 50(8): 1815-1819. https://doi.org/10.1007/s11250018-1623-6

Govindarajan R, Koteeswaran A, Venugopalan AT, Shyam G, Shaouna S, Shaila MS, Ramachandran S (1997) Isolation of pestes des petits ruminants virus from an outbreak in Indian buffalo (Bubalus bubalis). Vet Rec 141:573-574

Hashmi MM, Frate L, Nizami SM, Carranza ML (2017) Assessing transhumance corridors on high mountain environments by least cost path analysis: the case of yak herds in Gilgit-Baltistan, Pakistan. Environ Monit Assess 189:488-489. https://doi.org/10.1007/ s10661-017-6189-7

Khan R (1996) Yak production and genetic diversity in Pakistan. In: Miller D.G., Craig S.R. and Rana G.M. (eds), Proceedings of a workshop on conservation and management of yak genetic diversity held at ICIMOD, Kathmandu, Nepal, 29-31 October 1996.
ICIMOD (International Centre for Integrated Mountain Development), Kathmandu, Nepal. pp. 57-60

Kumar N, Maherchandani S, Kashyap SK, Singh SV, Sharma S, Chaubey KK, Ly H (2014) Peste des petits ruminants virus infection of small ruminants: a comprehensive review. Viruses 6:2287-2327. https://doi.org/10.3390/v6062287

Kwiatek O et al (2007) Peste des petits ruminants (PPR) outbreak in Tajikistan. J Comp Pathol 136:111-119. https://doi.org/10.1016/j. jсpa.2006.12.002

Li XH et al (2018) Epidemiological investigation and risk factors of Peste des petits ruminants (PPR) in yaks (Bos grunniens) and cattle in five regions of China. Trop Biomed 35:736-743

Libeau G, Prehaud C, Lancelot R, Colas F, Guerre L, Bishop DH, Diallo A (1995) Development of a competitive ELISA for detecting antibodies to the peste des petits ruminants virus using a recombinant nucleoprotein. Res Vet Sci 58:50-55

Liu F, Li J, Li L, Liu Y, Wu X, Wang Z (2018) Peste des petits ruminants in China since its first outbreak in 2007: a 10-year review. Transbound Emerg Dis 65:638-648. https://doi.org/10.1111/tbed.12808

Munir M (2014) Role of wild small ruminants in the epidemiology of peste des petits ruminants. Transbound Emerg Dis 61:411-424. https://doi.org/10.1111/tbed.12052

Shonazarov J (2012) Epizootological monitoring and improvement of measures to combat peste des petits ruminants in the Republic of Tajikistan

Zahur AB et al (2014) Epidemiological analysis of Peste des petits ruminants (PPR) outbreaks in Pakistan. J Biosci Med 2:18-26. https://doi.org/10.4236/jbm.2014.26004

Zhao W-H, Yang S-B, Han J-Q, Jiang M, Li H-C, Zhang N-Z, Li Q-H (2009) Confirmed diagnosis by RT-PCR and phylogenetic analysis of peste des petits ruminants virus in Tibet, China. Virol Sin 24:573578. https://doi.org/10.1007/s12250-009-3064-x

Publisher's note Springer Nature remains neutral with regard to jurisdictional claims in published maps and institutional affiliations. 